\title{
Le jardin collectif urbain : Un projet éducatif holistique et fondamentalement politique
}

Anne-Marie Legault

\author{
(2) OpenEdition \\ Journals \\ Édition électronique \\ URL : http://journals.openedition.org/ere/1545 \\ DOI : $10.4000 /$ ere. 1545 \\ ISSN : 2561-2271 \\ Éditeur \\ Centr'ERE
}

Référence électronique

Anne-Marie Legault, « Le jardin collectif urbain : Un projet éducatif holistique et fondamentalement politique », Éducation relative à l'environnement [En ligne], Volume 9 | 2011, mis en ligne le 20 décembre 2011, consulté le 21 février 2020. URL : http://journals.openedition.org/ere/1545 ; DOI : 10.4000/ere. 1545

Ce document a été généré automatiquement le 21 février 2020. 


\title{
Le jardin collectif urbain : Un projet éducatif holistique et fondamentalement politique
}

\author{
Anne-Marie Legault
}

1 Cet article présente une analyse des principaux résultats d'une étude exploratoire réalisée dans le cadre d'un projet de recherche de la Chaire de recherche du Canada en éducation relative à l'environnement (ERE) de l'Université du Québec à Montréal (UQAM). Portant sur l'éducation relative à l'éco-alimentation en contexte de jardinage collectif urbain, il est basé sur deux études de cas réalisées en collaboration avec des responsables de projets de jardins situés dans des quartiers aux réalités contrastées de Montréal.

2 Afin de bien comprendre l'intérêt de cette recherche, il convient d'ouvrir une parenthèse pour définir le concept de jardin collectif qui est au cœur de notre étude. Souvent définis par opposition aux jardins communautaires, qui sont composés de parcelles cultivées individuellement et regroupées côte à côte, les jardins collectifs sont des potagers cultivés collectivement par un groupe de jardiniers. Initiatives en effervescence à Montréal comme dans d'autres métropoles dans le monde, ils sont la plupart du temps portés par des organismes communautaires qui leur donnent une mission sociale variant selon les besoins des quartiers dans lesquels ils sont ancrés. L'ensemble des tâches reliées à l'entretien du jardin se déroule en groupe et la récolte est divisée entre les participants. Une partie de la récolte est parfois distribuée à une banque alimentaire ou à un autre organisme sociocommunautaire (Boulianne, 2001; Mahuziès-Sanuy, 2007 ; Courville, 2008 ; Duchemin, 2009). Un animateur socio-horticole représentant l'organisme porteur est souvent présent lors des séances de jardinage de groupe afin d'aider au bon déroulement de l'expérience et de veiller à l'atteinte des objectifs fixés par l'organisme.

Même si l'éducation est au cœur de la mission de nombreux projets de jardinage urbain (Duchemin et al. 2009), aucune recherche n'a encore exploré le potentiel éducatif de ces initiatives en milieu communautaire. L'éducation relative à l'éco-alimentation en 
contexte de jardinage collectif urbain est encore très peu systématisée, nourrie principalement de l'expérience et de l'intuition des porteurs de projets (Sauvé, 2006). Nous avons donc entamé cette recherche afin de contribuer à enrichir et valoriser ces initiatives du milieu éducatif non formel ${ }^{1}$.

Dans cet article, nous mettons l'emphase sur l'un des objectifs spécifiques de notre recherche, soit la caractérisation des dimensions critique, éthique et politique de l'éducation relative à l'éco-alimentation en contexte de jardinage collectif urbain. Nous présentons des éléments du cadre théorique de notre recherche ainsi qu'une analyse de certains résultats empiriques afin de faire un tour d'horizon de l'objectif de notre étude mentionné ci-haut. Par cette exploration, nous espérons contribuer à enrichir les dimensions critique et politique du champ de l'ERE.

Il nous semble difficile d'aborder l'éducation en contexte de jardinage collectif urbain sans situer l'émergence et l'effervescence de ce type de projets dans le phénomène plus large de l'agriculture en ville. C'est pourquoi, nous commençons cet article en posant un regard typologique sur le concept d'agriculture urbaine (AU) et en présentant un historique de l'évolution de sa manifestation dans le monde, mais surtout à Montréal. Nous abordons ensuite les problématiques sous-jacentes à la déterritorialisation de l'alimentation et expliquons l'intérêt que revêt une éducation axée sur le lien direct existant entre les humains et les écosystèmes qui les nourrissent. Nous poursuivons ce texte avec une définition de quelques concepts clés, essentiels à la compréhension des résultats de cette étude. Finalement, nous enchaînons avec des éléments conceptuels mettant en lumière les dimensions politique et critique de ce type d'initiatives. Nous établissons des ponts entre les données obtenues à travers nos deux études de cas et les éléments théoriques présentés.

\section{De l'agriculture au cœur des villes ? Une petite histoire}

Les mots "agriculture » et "urbanité » peuvent sembler, au premier abord, ne pas naturellement faire bon ménage. Formé à partir de deux mots latins, "ager» qui signifie « champ » et «cultura » qui désigne « culture » et faisant référence à l'idée de «cultiver », le concept d'" agri-culture » signifie, selon son étymologie, « la culture des champs ». Ainsi, parler de cultiver des champs ou autre espace afin de produire des denrées nourricières dans nos métropoles contemporaines peut aujourd'hui sembler une utopie. En effet, pour la majorité des citadins, l'agriculture est généralement reconnue comme une activité périurbaine associée à la campagne ou aux régions.

7 Pourtant, il importe de rappeler qu'à leurs origines, plusieurs cités intégraient des espaces agricoles au sein même de leur territoire afin de répondre aux besoins de leurs résidents. La région de Montréal, devenue aujourd'hui une agglomération de près de 2 millions d'habitants (Ville de Montréal, 2006), compte parmi les terres les plus productives du Québec (Ressources naturelles Canada, 2008). Toutefois, l'accroissement de la population, de la taille et des activités des villes eut comme impact d'éloigner la production alimentaire et de remplacer des hectares de terres arables cultivées par des développements résidentiels.

8 De nos jours, pour répondre aux besoins alimentaires des citoyens, les zones urbanisées dépendent de complexes réseaux de production, de transformation et de distribution basés sur des chaines de transport motorisées allant jusqu'aux quatre coins de la planète. Selon une étude du Worldwatch Institute, le trajet moyen parcouru par les 
denrées nourricières, du champ à la table, est de $2500 \mathrm{~km}$ (Halweil, 2002). Ainsi, les citadins n'ont jamais été aussi déconnectés de leurs sources alimentaires. Parmi les effets pervers de ce déracinement alimentaire, on retrouve la disparition des savoirs et savoir-faire agricoles et culinaires, transmis jusqu'alors de génération en génération, au profit de produits transformés et prêts-à-manger. À cette perte de culture et de compétences alimentaires s'ajoute une série d'impacts sur la santé des écosystèmes (pollution de l'eau et de l'air, perte de biodiversité, OGM, etc.) et sur celle de l'humanité (malnutrition, cancer, maladies cardio-vasculaires, inégalités, pauvreté, etc.).

\section{L'éducation à l'éco-alimentation pour renouer avec l'alimentation}

De plus en plus conscients des conséquences multiples de ce système mondial de ravitaillement déterritorialisé, plusieurs citoyens cherchent aujourd'hui des alternatives afin d'effectuer un virage vers une éco-alimentation. Défini par Lucie Sauvé (2006) comme "une alimentation saine (diversifiée et sécuritaire), produite, distribuée et consommée dans le respect des processus écologiques et de l'équité des rapports sociaux ", le modèle " éco-alimentaire " prend en considération les liens qui unissent l'alimentation, la santé, l'environnement et les rapports sociaux. L'éducation relative à l'éco-alimentation, axée ainsi sur le lien direct existant entre les humains et leurs écosystèmes, apparaît comme l'une des avenues essentielles à favoriser pour amener un changement radical de culture alimentaire et l'émergence de systèmes de production et d'approvisionnement alternatifs.

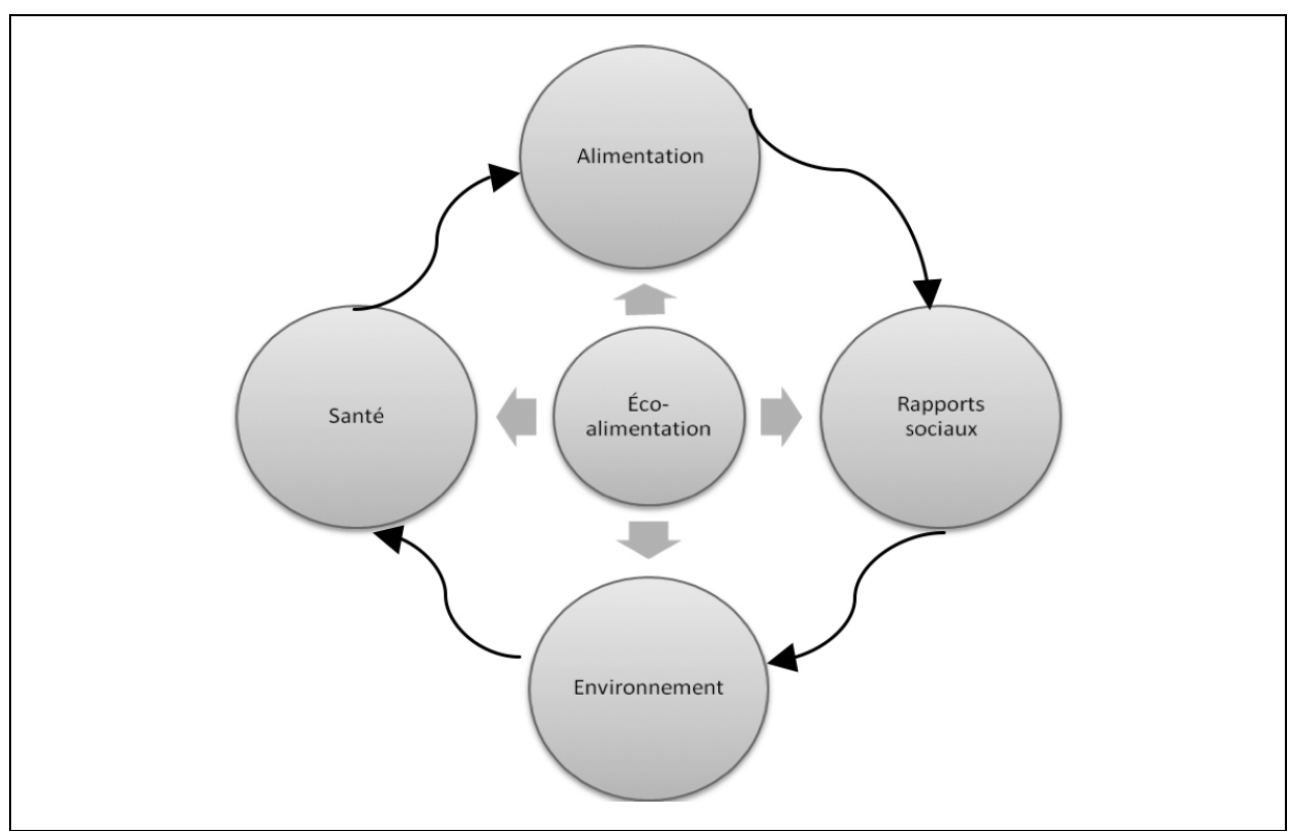

Figure 1 : L'éco-alimentation au carrefour de l'alimentation, de la santé, de l'environnement et des rapports sociaux. 


\section{Le caractère politique des choix alimentaires}

10 De plus en plus d'auteurs abordent les choix alimentaires en tant que gestes politiques (McMichael, 2001; Nestle, 2003 ; Pollan, 2006; Sauvé, 2009). Ils soulignent que chaque repas nous lie à l'environnement et à des milliers de personnes qui cultivent, récoltent, transforment, emballent et vendent notre nourriture. Ainsi, comme le mentionne Lucie Sauvé (2009) dans son article Le rapport entre éthique et politique : un enjeu pour l'éducation relative à l'environnement, «nos choix alimentaires portent un message : quand j'achète et consomme tel ou tel type d'aliments, j'encourage un certain système de production et de distribution. Manger devient un acte à dimension éthique et politique ». Si nous devenons conscients de la dimension politique de nos choix, nos habitudes alimentaires deviennent le reflet d'une série de valeurs et de considérations éthiques qui guident nos prises de décisions quotidiennes. Ces valeurs et décisions éthiques constituent dès lors les bases de notre relation politique et environnementale au monde. Toujours selon Sauvé (2009), ces dernières ne sont pas acquises une fois pour toutes, mais se construisent et se reconstruisent au quotidien. Dans le but de stimuler et de guider la formation de ces bases identitaires, l'ERE devient donc un processus à privilégier afin d'encourager le développement de savoirs, de savoir-faire critiques, et de savoir-être pouvant encourager l'adoption d'un vouloir agir en matière d'éco-alimentation (ibid.).

\section{De l'agriculture et des jardins collectifs en ville}

11 À Montréal, comme dans d'autres grandes villes du monde, l'agriculture urbaine est l'une des initiatives qui a émergé en tant que mouvement citoyen de réappropriation de l'espace et de l'alimentation en milieu urbain (Stiegman, 2004). Comprise dans sa définition la plus large comme la production alimentaire en milieu urbain, l'AU englobe une diversité d'activités prenant place dans les limites des agglomérations urbaines : la production de légumes, de fruits, d'herbes, de fleurs, de champignons, l'élevage d'animaux, l'apiculture, les activités de production forestière et même, parfois, la transformation et la vente des produits de ces activités dans les villes (Egziabher, 1994, in Boulianne, 1999, p.1). En effet, aujourd'hui plus de 800 millions de personnes pratiqueraient l'AU à travers le monde (Smit et al., 1996, in Duchemin et al., 2009).

Dans les pays les plus défavorisés, ce phénomène n'a rien de nouveau, l'AU y étant souvent une question de survie. Depuis le début des années 1970, les agences internationales d'aide au développement ont reconnu l'apport utile de l'agriculture urbaine à la sécurité alimentaire des populations démunies (Chevrier, 2001). Dans les pays riches, les citoyens sont aussi de plus en plus nombreux à s'adonner à la culture nourricière en pleine ville. Selon Henning (1997), ces citadins cultivent des légumes non tant par nécessité, mais plutôt pour combler des besoins que l'agriculture industrialisée ne peut satisfaire : par plaisir, pour sympathiser avec ses voisins ou pour se garantir des aliments sains et sans intrants chimiques. La pauvreté n'étant pas absente des villes des pays du Nord, la culture potagère vient parfois suppléer à l'alimentation des foyers démunis (Stiegman, 2004). Peu importe les motivations, le nombre de jardiniers urbains est en augmentation constante dans les grandes villes des pays industrialisés.

13 La ville de Montréal n'échappe pas à cet engouement pour la culture potagère urbaine. Aujourd'hui, plus de 15000 jardiniers profiteraient du programme public de jardinage 
communautaire de la Ville mis en place dans les années $70^{2}$ qui permet aux habitants de la ville d'avoir des jardinets (Duchemin et al., 2009). Compte tenu du nombre insuffisant de parcelles disponibles, les jardins de type collectif sont alors apparus dans plusieurs quartiers de la métropole afin de répondre à la demande croissante des citoyens (Hista, 2007) et de contrer à la situation économique difficile (années 90). On dénombre aujourd'hui plus d'une quarantaine d'initiatives réparties dans une dizaine de quartiers montréalais (Nourrir Montréal, 2009). Plus de 2000 personnes y jardinent (Lebedeva, 2008) et ce nombre est en constante augmentation depuis quelques années (Nourrir Montréal, 2009).

\section{Éléments de méthodologie}

14 Notre recherche porte sur le potentiel éducatif des projets de jardinage collectif. L'éducation est au cœur de la mission de la plupart de ces projets (Duchemin et al., 2009) et ces initiatives deviennent de plus en plus importantes dans le paysage des alternatives éco-alimentaires. Afin de saisir le phénomène en profondeur, nous avons réalisé deux études de cas, en collaboration avec des organismes porteurs de projets de potagers collectifs situés dans des quartiers aux réalités sociodémographiques contrastées, soit les secteurs Villeray et Notre-Dame-de-Grâce (NDG). Comme chaque jardin est le reflet unique du milieu auquel il appartient (Stiegman, 2004, p. 18), cette collaboration avec deux organismes avait comme objectif de rendre compte de l'éventail et de la richesse des possibilités d'intervention éducative en contexte de jardinage collectif urbain.

15 Tout au long de notre démarche, nous avons tenté de mettre en lumière les fondements, les pratiques, les enjeux et les dimensions politique et critique de ces projets d'éducation à l'éco-alimentation. Pour ce faire, nous avons utilisé plusieurs stratégies de cueillette de données, telles l'analyse documentaire, des entrevues semidirigées et de groupe ainsi que l'observation participante. Les données colligées ont été analysées selon une « logique inductive et délibératoire " (Lessard-Hébert et al., 1994) suivant les thèmes et objectifs élaborés en début de recherche, mais aussi de manière à laisser émerger de nouvelles dimensions non prévues au départ, lorsque ces dernières étaient plus riches que le cadre préalablement établi.

Dans les différentes sections, nous présentons certains résultats de notre démarche, en mettant une emphase particulière sur les dimensions critique et politique du phénomène étudié. Nous présentons d'abord des éléments récoltés lors de notre revue de littérature, nous permettant de définir les concepts clés de cette recherche et d'identifier les dimensions politique et critique pouvant s'appliquer au jardinage collectif urbain. Nous accordons une attention particulière aux écrits qui traitent également de leur dimension éducative. Tout au long des prochaines sections, nous avons inséré des éléments de résultats empiriques qui viennent appuyer, complémenter et bonifier les données théoriques présentées. 


\section{Cadre théorique : définition du concept de « chose politique »}

17 Nous suggérons ici une vision beaucoup plus large du concept que celle généralement véhiculée. Nous considérons que le politique peut dépasser le cadre de l'appareil législatif et représentatif qu'est l'État et aller au-delà de la participation «traditionnelle » (exercice du droit de vote, militance au sein d'un parti politique) à ce système politique.

Afin de mieux en comprendre le sens, nous commençons par approfondir l'origine du concept qui nous intéresse. Selon ses racines étymologiques, le mot politique vient du latin « politicus», et du grec « polis» qui signifie ville ou cité. Ainsi, dans sa définition la plus simple, le politique concerne tout ce qui a trait aux affaires publiques, aux affaires de la cité, ou ce qui intéresse les politai (citoyens), acteurs de la vie politique. De ce point de vue, le politique est bien loin de représenter seulement les structures ou fonctions de pouvoir et de gestion des sociétés.

Selon Swartz (1968, p. 1), le politique peut aussi se rapporter «à des évènements qui ont trait à la détermination ou à la réalisation d'objectifs publics et/ou à la distribution différentielle (du pouvoir), ainsi qu'à l'utilisation du pouvoir dans le ou les groupes intéressés par ces objectifs » (trad. libre). Chaia Heller, figure importante du courant de l'écologie sociale, pousse le concept plus loin. Dans son ouvrage Désir, nature et société, elle définit le politique comme un acte à la portée de tous, pouvant commencer par des conversations intéressées: "L'activité politique est ce qui se passe quand les citoyens se réunissent pour discuter, débattre et décider les mesures politiques qui conditionneront leur existence en tant qu'habitants d'une ville ou d'un village " (Heller, 2002, p. 216). Cette vision du politique comme "sphère d'activités non partisanes » (Sauvé, 2009, p. 154) met l'emphase moins sur les institutions, les fonctions ou les structures que sur des processus politiques reposant sur l'action. Cette action dite politique peut prendre différentes formes, mais doit chercher à agir sur la direction des affaires publiques dans un domaine ou un autre de la vie communautaire (Breton, 1983). Ainsi, si Heller qualifie de politique une réunion de citoyens soucieux de leur milieu et que le récent mouvement pour une consommation responsable fait de la consommation un geste politique avec le slogan "acheter, c'est voter », un projet de jardinage urbain ne pourrait-il pas lui aussi avoir une dimension politique?

\section{Le jardin présenté comme espace éducatif à l'image du monde}

Dans sa plus simple expression, le jardin signifie le lieu où l'on cultive des végétaux (Mahuziès-Sanuy, 2007, p. 16). Philippe Mahuziès-Sanuy, qui s'est intéressé au potentiel pédagogique du jardin et du jardinage pour la formation continue des enseignants au primaire, présente le jardin comme un objet et un contexte de formation pertinent favorisant des stratégies pédagogiques qui tiennent davantage compte du rapport au monde (2007, p. xi). Afin de mieux comprendre la multiplicité des formes, des fonctions et des sens attribués au jardin et au jardinage, ainsi que de clarifier les nombreuses représentations et valeurs sous-jacentes exprimées par les participants à son étude, l'auteur a élaboré une carte conceptuelle du jardin (figure 2). Il a croisé les différents 
types de jardins évoqués par les participants avec la typologie et les valeurs associées aux différentes représentations de l'environnement proposées par Sauvé (1997, p. 11-15)

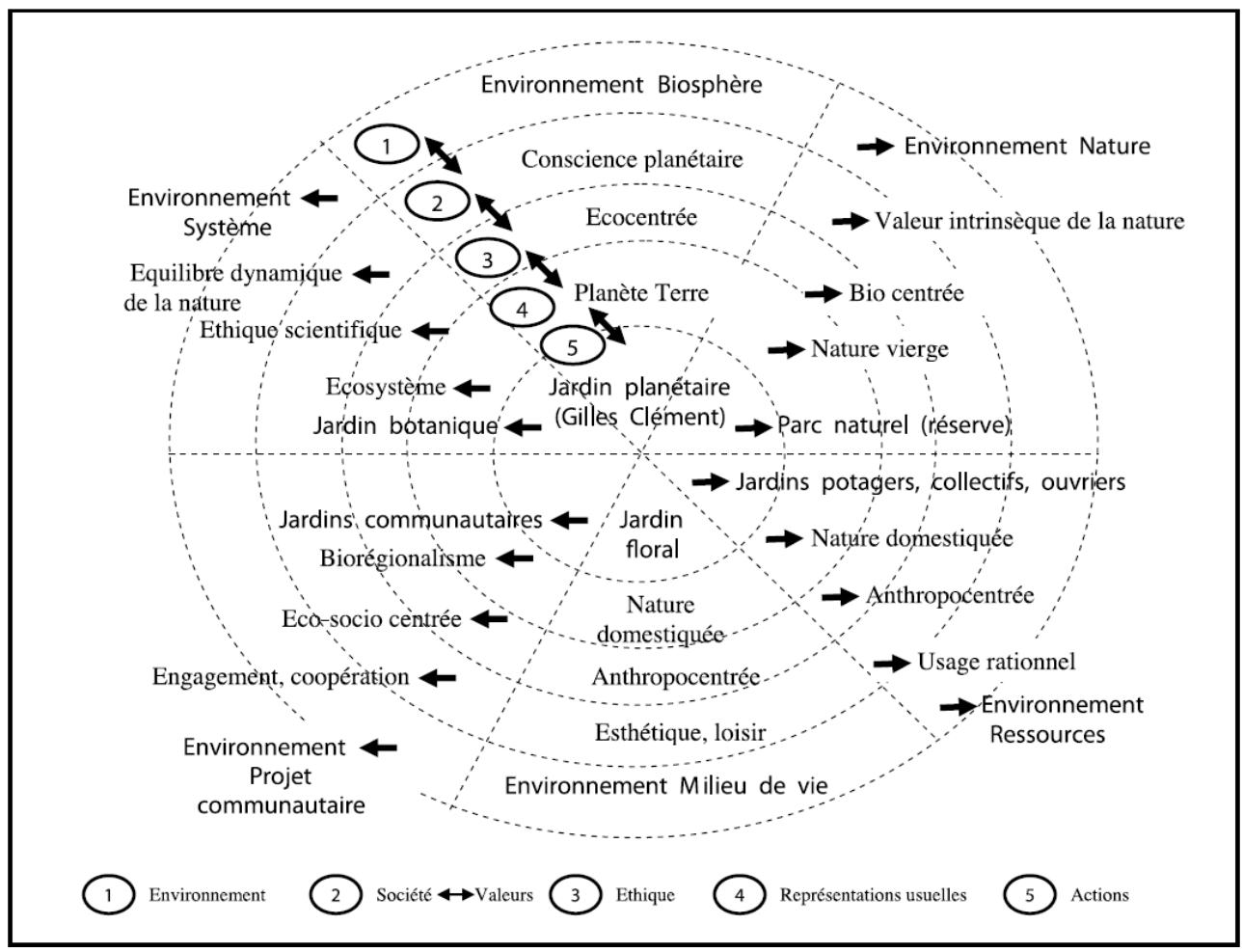

Figure 2 : Carte conceptuelle du jardin selon Mahuziès-Sanuy (2007, p. 24) comme projet éducatif et l'environnement, nous avons observé que toutes les visions présentées par Mahuziès-Sanuy ont été évoquées par les participants à l'étude. Plus particulièrement, nous avons noté que les projets de jardin collectif étudiés présentent fréquemment une réalité plus globale et holistique que celle qui apparaît à première vue, rappelant la représentation d'environnement-biosphère proposée par MahuzièsSanuy.

Cette vision du jardin ne serait pas récente. En effet, Michel Foucault, dans une conférence donnée à un groupe d'architectes et intitulée Des espaces autres en 1967, décrivait le jardin comme "la plus petite parcelle du monde et puis la totalité du monde ». Il disait alors: "le jardin c'est, depuis le fond de l'Antiquité, une sorte d' hétérotopie $e^{3}$ heureuse et universalisante", ayant le pouvoir de "juxtaposer en un seul lieu réel plusieurs espaces, plusieurs emplacements qui sont en eux-mêmes incompatibles » (Foucault, 1967). Selon cette représentation, le jardin renvoie autant à des éléments du microcosme que du macrocosme (Brunon et Mosser, 2007). MahuzièsSanuy (2009, p. 231) ajoute que la dimension pédagogique et éducative du jardin "réside dans le fait qu'il est possible d'une part d'intervenir sur ce milieu et d'y observer, à petite échelle, les interactions des milieux plus vastes, et d'autre part, d'ouvrir une réflexion sur notre rapport au monde». Il est un objet de formation pertinent et porteur en ERE car il favorise la mise en œuvre «de stratégies pédagogiques qui tiennent compte du rapport au monde, de la globalité et de la complexité de l'objet d'apprentissage (réseau de relations personne - société - 
environnement) »(Mahuziès-Sanuy, 2007, p. 1). Ainsi, qu'il soit petit ou grand, clôturé ou sans balise physique, le jardin, comme environnement cultivé par l'humain, peut être envisagé comme une allégorie du monde actuel.

C'est en suivant cette réflexion que le maintenant célèbre paysagiste français Gilles Clément ${ }^{4}$ faisait en 1999 une exposition sous le thème du Jardin planétaire, concept représentant la planète comme un jardin. Dans son Manifeste du Tiers Paysage (2004, p. 2), Clément explique sa réflexion en nous ramenant à l'étymologie du mot jardin, qui vient de "garten", un enclos dans lequel on protège le meilleur: le meilleur des plantes, le meilleur de l'espace, le meilleur de la lumière, etc. Selon Clément, comme nous sommes aujourd'hui conscients de la finitude écologique de la planète et des « limites de la biosphère comme l'enclos du vivant» (ibid., p. 3), le jardin devient un espace pour protéger ce que l'humanité a de meilleur, c'est-à-dire la diversité et la Vie, au sens large. Selon cette vision, le jardin devient un lieu où les citoyens peuvent apprendre à observer ces deux éléments indissociables et à clarifier, en tant que jardiniers planétaires, leur relation à l'environnement et au monde. Pour ce paysagiste engagé, le jardin est donc un terreau fertile d'apprentissages permettant de réfléchir aux modes de gestion collective de la planète et encourageant une refonte de l'art de vivre et du rapport de l'homme à son environnement social et biologique (ibid., p. 3).

\section{Le jardin comme projet collectif au cœur de la ville : un interstice biopolitique}

Lorsqu'on entre dans un jardin collectif situé au centre de la ville, on se sent tout de suite dans un monde à part. Une oasis de verdure et d'odeurs au cœur d'un paysage minéralisé, un riche éco-sociosystème (Goffin, 1999) au milieu de celui de la métropole, un espace où des citoyens se rassemblent afin de transformer leur environnement autour d'un projet commun. Pour certains auteurs, le jardin devient le symbole d'une résistance de la vie, une critique de sens et de rationalité à dimension politique dans la métropole.

En donnant l'exemple d'ÉCObox ${ }^{5}$, un jardin partagé du quartier de La Chapelle dans le 18e arrondissement de Paris, les architectes Constantin Petcou et Doina Petrescu (2008) parlent des jardins urbains gérés collectivement comme interstices ou espaces biopolitiques (espaces de résistance au biopouvoir) dans la ville où les phénomènes de reproduction de la vie organisée (sociale, politique) sont contrôlés, captés et exploités selon les règles de la société capitaliste (Negri, 2008, p. 19). Ces interstices ou espaces biopolitiques deviennent des lieux de résistance de la vie (ou du bio) pouvant servir d'endroits "de dés-apprentissage des usages assujettis au capitalisme et de réapprentissage d'usages singularisés, en produisant une subjectivité collective et spatiale propre aux sujets investis" (Petcou et Petrecu, 2008, p. 106). Partant des réflexions de Foucault sur le jardin comme espace de l'autre, Petcou et Petrescu proposent de parler d'alterotopiques, c'est-à-dire «d'espaces construits et partagés "avec les autres", avec ceux qui diffèrent de nous et qui nous importent » (ibid., p. 106), devenant ainsi des espaces de l'agir. Dans ces lieux, des citoyens se regroupent et agissent ensemble par la prise en charge d'un projet collectif, d'un jardin.

Citant les travaux de Jacques Rancière (1998), ces mêmes auteurs notent que le collectif favorise l'apparition « d'un sujet qui se pense par rapport aux autres », permettant aux 
participants de se recréer une identité subjective différente de celle valorisée dans le milieu social et professionnel capitaliste (emploi, statut social, etc.). La prise en charge d'un jardin en groupe peut permettre d'adopter des positions, des rôles nouveaux, d'engendrer des subjectivités que les participants se construisent entre eux. De ces nouvelles subjectivités peuvent naître des dimensions politiques non prévues au départ. Toni Negri (2008), dans un article issu d'une discussion avec les architectes Petcou, Petrescu et Querrien, parle d'un activisme au quotidien, pouvant commencer par la résistance à la société de consommation, et une volonté de se réapproprier des fonctions essentielles à la vie quotidienne comme l'alimentation ou le logement, etc. Ces architectes mentionnent toutefois que, selon eux, l'action politique n'est pas naturelle, allant bien au-delà de la socialisation qui peut s'apprendre plus facilement. Ils soulignent :

Le politique (...), c'est de la citoyenneté, de la démocratie, de l'égalité. La subjectivité, le pré-individuel, c'est une sorte de condition pré-politique. Pour pouvoir agir politiquement, il faut déjà être quelque part... [Les espaces biopolitiques] permettent d'accueillir l'émergence des subjectivités et d'aller plus loin après, si c'est possible. (Negri, 2008, p. 21)

Ainsi, pour ces auteurs, le jardin collectif au cœur de la ville devient l'un de ces espaces urbains permettant l'émergence de ces nouvelles subjectivités et la (re)construction de ce que Mitchel Thomashow (1995, p.105) appelle l'identité politique. Cette identité politique est intimement liée à l'identité écologique à travers le concept d'écocitoyenneté. Par une démarche praxique (réflexion sur l'action) ancrée dans le quotidien, le professeur Thomashow invite ses étudiants à prendre conscience de leur relation politique au monde et à (re)construire consciemment cette dernière.

Dans notre étude, nous avons tenté d'observer si le jardin collectif urbain, comme projet d'éducation relative à l'environnement axé sur l'alimentation, peut être un contexte de prises de conscience face à cette identité politique, soit par les relations interpersonnelles, la façon dont les décisions de groupes sont prises, la manière dont la communication se déroule, etc. Selon Thomashow, (1995, p. 106), par une observation attentive des comportements sur une petite échelle, il devient possible de questionner notre rapport aux relations de pouvoirs en place. Le tout peut ensuite se transposer à un niveau plus macro, en observant les habitudes citoyennes (vote), la consommation (choix de produits), les choix éthiques, les valeurs, etc. Selon cette vision, le jardin, comme microcosme à l'image du monde, devient un espace propice pour clarifier et/ou resituer notre rapport au monde tant politique, écologique que social. L'éducation devient l'un des processus privilégiés pour accompagner ces questionnements ainsi que l'action sociale dans laquelle les jardiniers sont déjà, ou voudraient s'engager.

\section{La dimension politique du jardinage collectif urbain}

Même si la plupart des participants rencontrés n'ont pas directement fait référence à la dimension politique de leur expérience de jardinage collectif urbain et que ceux à qui nous avons posé la question nous ont souligné leur peur de marginaliser encore plus ce type d'initiatives en y associant une telle dimension, plusieurs des personnes rencontrées nous ont fait part d'un cheminement personnel et social, attribuable à leur participation à un projet de jardin collectif. Que ce soit au niveau de leur santé, de leurs habitudes alimentaires, de leur socialisation, de leur relation à l'environnement, de leur lien à la communauté, les impacts et retombées de leur participation à un projet de 
jardin collectif sont multiples. Pris séparément, ils peuvent sembler minimes, mais comme nous l'explique France Sainte-Marie, une jardinière de Villeray donnant également des formations pour l'organisme, il est difficile de prédire à long terme les répercussions globales de tous ces petits changements :

Si tu te sens mieux dans ta peau, si tu as plus confiance en toi et que tu parles à des gens à qui tu n'aurais pas parlé avant, si tu essaies de nouveaux aliments et que tu t'ouvres à d'autres choses, tu nourris automatiquement ton estime de toi. Si tu nourris ton estime de toi, un petit pas en amène un autre, qui en amène un autre. Et qui sait si des gens qui ne travaillaient pas depuis plusieurs années peuvent se trouver un travail à temps partiel. Cela peut faire boule de neige. Donc en partant de l'éducation, socialement c'est un projet qui, sur le long terme, peut inciter directement les gens à travailler, il peut contribuer à la réduction du taux de chômage, à la diminution du nombre d'assistés sociaux, à l'émergence d'une société plus en harmonie, plus à l'écoute des autres, plus respectueuse des coutumes des gens, des personnes qui apprennent à travailler. Travailler la terre, si tu veux avoir quelque chose, cela t'amène à travailler pour voir le fruit de ton travail, à être valorisé.

Les jardins amènent également toute une série de valeurs et de nouvelles références pour les participants. La coordonnatrice du réseau des jardins collectifs de Villeray, Magdouda Oudjit, souligne, par exemple, l'importance de ce type de projet dans l'apprentissage du vivre ensemble et des valeurs collectives :

Les jardins collectifs donnent une base importante pour l'éducation des personnes en changeant leur système de références individuelles à un système de références collectives. C'est assez difficile à réaliser, mais c'est un des moyens pour changer les habitudes des personnes, pour qu'elles puissent penser non seulement à ellesmêmes mais aussi aux autres.

31 Ainsi, comme les participants au projet semblent peu conscients de la dimension politique et parlent plutôt de changement social, nous avons choisi d'orienter davantage notre recherche vers la dimension critique de l'expérience.

\section{Le jardin collectif comme projet éducatif à dimension critique}

32 Lucie Sauvé et Isabel Orellana (2008, p. 7), soulignent que «le développement d'une compétence critique est l'un des objectifs majeurs de l'éducation relative à l'environnement». Elles rappellent que la visée de l'éducation relative à l'environnement est «l'émancipation, l'équité et la justice sociale » en mettant à jour les relations de pouvoir afin de contrer les oppressions et l'aliénation (ibid., p. 8). Dans ce sens, la dimension critique de l'ERE (ou criticité) est étroitement liée à la dimension politique de celle-ci (ibid., p. 10). Telle la dimension politique, la posture critique n'est pas seulement théorique, mais fait appel à « l'engagement de l'être dans l'action » (ibid., p. 9), en toute cohérence avec ses valeurs et son éthique.

33 Dans les projets de jardins collectifs étudiés, plusieurs des participants rencontrés se sont dits conscients du caractère critique et à contre-courant de l'expérience de jardinage collectif urbain. Pour n'en citer que quelques-uns, un jardinier de Villeray, Alain Carmel, souligne qu'à la base jardiner en ville est une expérience marginale :

Les jardins collectifs poussent plus loin l'idée de marginalité parce que nous [y] faisons du jardinage en ville, dans l'un des quartiers les plus urbanisés de Montréal. 
Cela détonne et nous met un petit peu plus en marge. Mais mine de rien, cela ajoute à la dynamique, à la façon de penser et à la façon de faire.

l'objectif d'empowerment qui sous-tend les expériences de jardinage collectif observées. Bien que le concept d'empowerment ait été théorisé par de nombreux auteurs, pour cette recherche nous nous référons particulièrement aux écrits d'Hippolyte qui a effectué une recension d'écrits très exhaustive sur le sujet. À partir de cette dernière, l'auteur a identifié sept dimensions au concept :

1. l'empowerment est un concept positif, car il met l'accent sur les forces des individus et vise leur bien-être ;

2. l'empowerment est un concept dynamique, car il est un processus ;

3. l'empowerment est un concept de l'agir, car l'idée d'action y est intrinsèque ;

4. l'empowerment est un concept dialectique, car il véhicule plusieurs paradoxes ;

5. l'empowerment est un concept écologique, car il oblige à prendre en compte les liens entre l'individu et l'environnement;

6. l'empowerment est un concept transformateur, car il vise des changements sociaux et politiques ;

7. l'empowerment est un concept politique, car l'idée de pouvoir est centrale à celui-ci. (Hyppolite, 2002, in Courville, 2008, p. 25-26)

Nous avons ajouté une huitième dimension à ce concept, soit l'empowerment comme processus éducatif, car il implique des apprentissages (acquisition de savoirs, savoirfaire et savoir-être) critiques et émancipatoires.

Autant dans l'initiative de Villeray que dans celle de NDG, nous avons observé que l'implication et la prise en charge d'un jardin collectif en groupe, dont le but est la récolte la nourriture de qualité, sont perçues comme un premier pas d'émancipation personnelle et d'implication dans la communauté. La coordonnatrice du réseau de jardins collectifs de NDG, Kelly Krauter, résume bien ce propos :

Nous voulons que les gens se sentent à l'aise et libres de s'impliquer, de vivre une vie démocratique, en prenant des décisions ensemble et en prenant conscience des effets de nos actions sur la Terre. On veut que les gens trouvent un intérêt à la vie communautaire et qu'ils tissent des liens entre eux. C'est vraiment une occasion pour eux de trouver un espace accueillant.

C'est aux animateurs présents dans le jardin que revient le rôle de veiller au bon déroulement $\mathrm{du}$ projet dans toute sa complexité, de soutenir l'engagement des participants et de coordonner la dynamique de groupe. Ils sont encouragés à appliquer des principes de co-gestion et de co-responsabilisation ${ }^{6}$ de l'animation afin de favoriser une prise en charge collective du jardin par les participants, dans une optique d' 
empowerment. Pour Kelly Krauter, la directrice d'Action Communiterre, "[L' empowerment] est probablement la partie du rôle de l'animatrice la plus importante ». Elle poursuit :

L'empowerment est de soutenir l'engagement, la participation des gens (...) sans tout leur donner. Il faut leur permettre de faire des erreurs pour qu'ils comprennent que l'on travaille ensemble et que ce n'est pas la fin du monde. On peut apprendre et la prochaine fois, agir différemment. Par exemple, si je vois un insecte sur une plante et que je demande à l'animatrice de l'identifier, elle devrait me suggérer un bouquin rangé dans le cabanon, en me demandant de le chercher pour le consulter et pour que nous puissions identifier cet insecte ensemble. Alors, c'est à moi de faire la démarche, de rechercher l'information, puis de partager ce que j'apprends avec les autres pour que la prochaine fois nous puissions l'identifier plus facilement. L'empowerment, c'est de fournir des outils, mais c'est aussi [aux participants] de découvrir comment s'en servir.

L'empowerment peut être ainsi considéré à la fois comme un but à atteindre et comme une approche à adopter. Dans le sens du célèbre pédagogue brésilien Paulo Freire ${ }^{7}$, l'éducation dans ces oasis collectives urbaines a une visée émancipatrice, en espérant que ce type de projet soit l'amorce pour inciter les jardiniers à développer le vouloiragir, à la base des pouvoirs, et les savoir-agir nécessaires à l'engagement et à la participation citoyenne.

La dimension critique des projets de jardins collectifs observés s'exprime souvent par le biais de l'approche conscientisante qui consiste à encourager le développement d'une réflexion critique par le dialogue avec les pairs. L'animateur tente de faire des liens entre le jardin et divers enjeux. L'une des stratégies souvent utilisée par les animateurs est d'amorcer des discussions amenant les jardiniers à réfléchir en groupe sur certains enjeux socioécologiques. Mohand Djebrani, animateur dans les jardins portés par la Maison de quartier Villeray, nous donnait un exemple :

J'essaie de faire passer des messages, (...) sur le compostage, sur la santé, sur ce que contiennent les produits de nos jardins comme valeur alimentaire. (...) Tout ça, ce n'est pas sous la forme d'un cours, on ne fait jamais de cours bien structuré, mais cela se fait au fur et à mesure. Chaque jour, il y a des discussions avec chaque groupe sur un volet, avec l'autre groupe, c'est autre chose. Cela dépend aussi des gens et de leurs préoccupations... Donc, selon la composante du groupe, on oriente les discussions. Mais, comme mentionné, ce ne sont pas des cours structurés. Ce sont plutôt des flèches : je lance un mot ou quelqu'un d'autre lance un mot et on discute autour de ces mots. On fait un cours sans le savoir.

Ainsi, grâce à des discussions spontanées ou planifiées, les jardiniers sont encouragés à dialoguer avec les autres participants afin de clarifier ou confronter leurs opinions et cadres de références sur différents enjeux. Pour certains, la discussion permet même de s'ouvrir et se questionner sur plusieurs dimensions de leur relation au monde et de faire des liens entre l'action locale et ses répercussions globales. Comme le confirme Anna Lee Popham, une animatrice dans un jardin de NDG : «Pour moi, il y a vraiment un lien entre un petit jardin, le jardin Thyme, et les problématiques [alimentaires] globales (...)... c'est une manière de travailler sur ces enjeux-là ». Elle poursuit :

[Le jardin collectif est l'occasion de] comprendre que beaucoup de nos aliments viennent de loin et contiennent de nombreux produits chimiques. Cette manière de nous alimenter est problématique parce que plusieurs personnes dans le monde ne sont pas assez nourries. Les jardiniers peuvent donc conclure eux-mêmes que ces inégalités sont assez bizarres. 
Même si l'alimentation est au cœur du projet éducatif porté par les jardins collectifs de notre étude, elle permet d'ouvrir sur de nombreuses autres dimensions de l'expérience.

\section{Un projet éducatif multidimensionnel favorisant une approche holistique}

Nombreux sont les auteurs qui se sont penchés sur les différentes sphères d'interventions, de fonctions, d'objectifs, et d'impacts des projets d'agriculture et de jardinage en milieu urbain (Boulianne, 1999; Henn, 2000 ; Boulianne, 2001 ; BouvierDaclon et Sénécal, 2001; Mougeot, 2006 ; Reyburn, 2006). Mais rares sont ceux qui ont affirmé le caractère multidimensionnel de telles initiatives ${ }^{8}$. En observant les projets de jardinage collectif sous l'angle éducatif, spécialement dans le cadre du présent article dans lequel nous nous intéressons plus spécifiquement aux dimensions critique et politique, il nous a semblé essentiel de mettre de l'avant cette multidimensionnalité afin de faire ressortir le potentiel holistique de ces initiatives. La figure 3 a été réalisée à partir d'une recension d'écrits et des propos recueillis lors des entrevues menées dans le cadre de notre recherche.

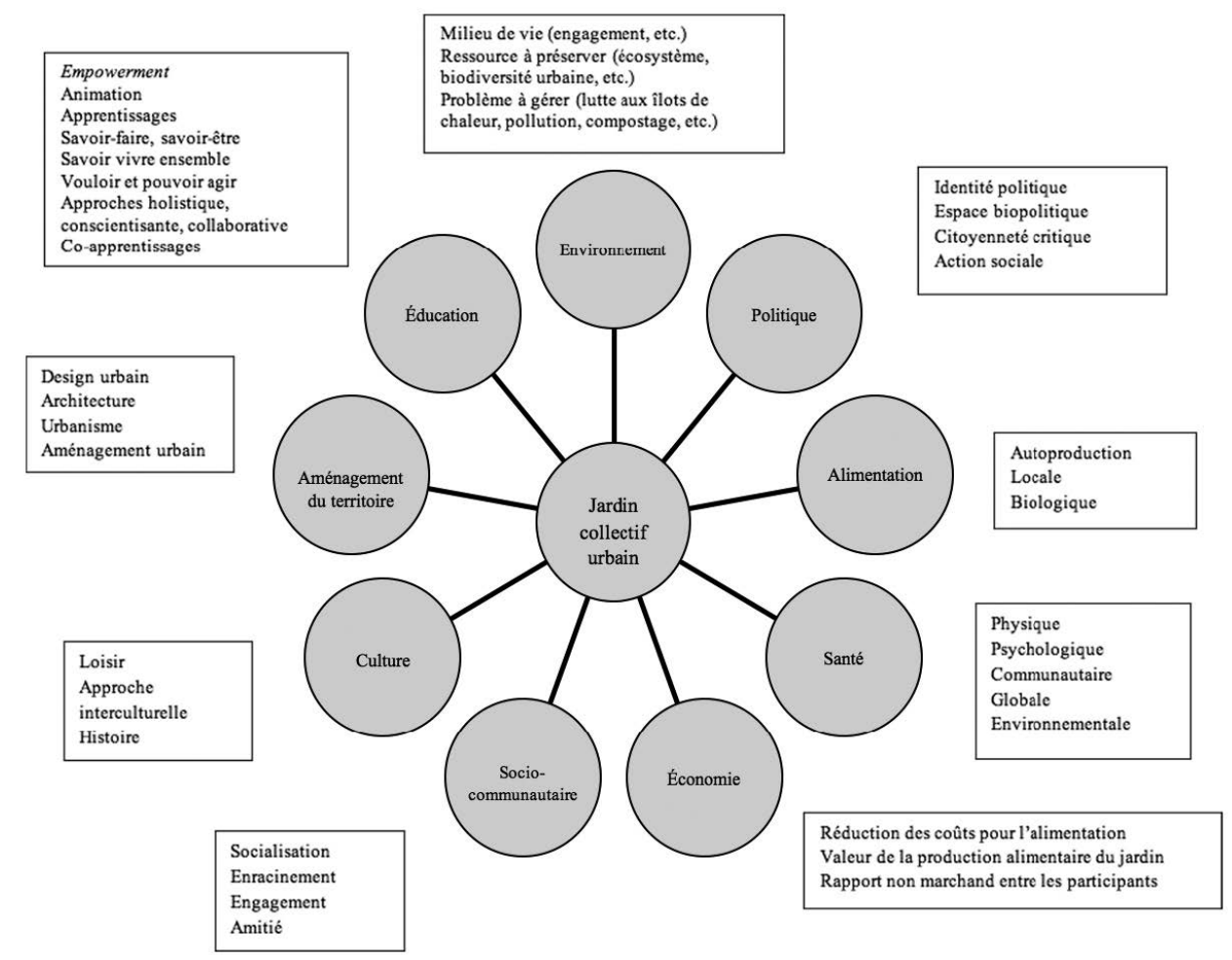

Figure 3 : Les multiples dimensions du jardin collectif urbain, inspiré de Duchemin et al. (2009)

Selon ce regard, le jardin collectif urbain comme projet éducatif et microsystème socioécologique peut représenter, de manière réduite, plusieurs dimensions de notre relation au monde. Même si ces différentes dimensions peuvent être prises en compte séparément comme cadre d'analyse de l'expérience de jardinage collectif urbain, elles sont toutefois indissociables les unes des autres dans l'expérience éducative par les apprentissages, les motivations et les savoirs développés tout au long de la saison de 
jardinage. Les apprentissages observés sont souvent transversaux à plus d'une dimension, contribuant ainsi au potentiel holistique de ce type de projet.

Par exemple, il est possible que, dans un jardin, l'animateur mette l'emphase sur l'hortithérapie ${ }^{9}$ lorsqu'il travaille avec un groupe de jardiniers ayant des problèmes de santé mentale, alors que, dans un autre jardin, il insistera davantage sur la production de légumes frais pour répondre aux besoins des personnes plus défavorisées. Ainsi, un jardinier peut venir au jardin principalement pour la socialisation et s'intéresser par la suite aux enjeux environnementaux par le biais de la problématique des déchets ou de l'expérimentation du compostage.

Il est à noter que deux réalités distinctes, mais interreliées, influencent l'expérience éducative vécue par les participants : celle de l'animateur responsable de l'atteinte des objectifs sociaux de l'organisme porteur du projet de jardin et celle du jardinier influencé par sa propre motivation et ses objectifs personnels à participer au projet. Le jardinier ne connaît pas nécessairement tous les objectifs de l'animateur et ce dernier n'est pas non plus toujours conscient des motivations des jardiniers.

Vécu comme un processus continu, fluide et évolutif, le processus éducatif appliqué au jardin collectif ne correspond pas au rapport traditionnel entre éducateur et éduqué. Il est nourri par les nombreuses interrelations à la base du projet : entre l'animateur et les jardiniers, les jardiniers et le jardin, le jardin et l'animateur, les individus et le groupe, le groupe et la communauté élargie, etc. Le projet éducatif des jardins collectifs de notre étude semble intimement lié à l'action - développement de savoirs et savoirfaire à travers des projets communs - et au lieu (ancrage local). Il apparaît donc s'inscrire dans les courants praxique - apprentissage dans et pour l'action tout en développant une compétence réflexive - et biorégionaliste - développement de compétences en matière d'éco-développement communautaire, local ou régional à travers des projets souvent portés collectivement - en éducation relative à l'environnement (Villemagne, 2005).

Selon les objectifs du projet, le jardin peut représenter autant le contexte de la situation éducative, un objet d'apprentissage, une stratégie, une approche qu'un but. Il sera un but lorsque l'objectif principal de l'activité est lié à la réalisation de ce dernier. Selon les principes de la pédagogie de projet, il peut également être utilisé comme prétexte à l'atteinte d'autres objectifs; on dira alors que celui-ci devient une stratégie pédagogique. Dans le courant de l'éco-formation, le jardin collectif sera utilisé comme contexte éducatif pour le contact avec la nature qu'il procure, devenant ainsi l'un des objets d'apprentissage et le milieu de la situation pédagogique.

Considérant toutes ces possibilités, le jardin collectif urbain se présente comme un projet éducatif au potentiel holistique permettant, grâce à son caractère multidimensionnel, d'aborder des réalités complexes et plurielles auxquelles nos sociétés doivent faire face aujourd'hui.

\section{Conclusion : le jardin collectif comme projet éducatif multidimensionnel à dimensions critique et politique}

Dans cet article, nous avons présenté une réflexion exploratoire sur les dimensions critique et politique de l'éducation à l'éco-alimentation dans les jardins collectifs urbains. Nous avons démontré que ces initiatives incitent à repenser la relation à 
l'environnement, à la santé et à la communauté. En impliquant les jardiniers directement dans la production de leur éco-alimentation, le jardin urbain permet des allers-retours entre les enjeux locaux et globaux, ainsi qu'une refonte à petite échelle des relations individus-environnement-société.

Même si les participants semblent peu conscients de la dimension politique de l'expérience, ces projets d'auto-production alimentaire à saveur sociale au cœur des villes portent cette dimension et permettent aux jardiniers de récolter beaucoup plus que des légumes. Ces projets sont, par exemple, des occasions de créer des liens avec d'autres résidents du quartier (social), d'apprendre à composter (environnement), de bouger (santé physique) et d'apprendre les rouages de l'agriculture biologique (alimentation). Ainsi, le jardin collectif urbain, en tant que micro-sociosytème à l'image du monde, devient un terreau éducatif fertile, où se déroulent de petites révolutions quotidiennes.

On peut rêver que de plus en plus de communautés et de quartiers soient contaminés par ces petits interstices biopolitiques, nourrissant autant les participants que leurs communautés. En tant que projets éducatifs au potentiel multidimensionnel, ils deviennent des refuges pour ce que Jérôme Clément (2004) appelle « le meilleur de la diversité de la Vie » en ville, encourageant de nouvelles relations de pouvoir entre les jardiniers et l'environnement, les jardiniers et la société, et les jardiniers entre eux. L'éducation à saveur critique et émancipatrice peut devenir, au cœur de ces oasis potagers, un moteur de changement politique, de construction de nouvelles subjectivités qui aideront à l'émergence d'un monde plus cohérent avec les valeurs des jardiniers, dans lequel l'économie serait subordonnée aux relations sociales et à l'environnement, un peu comme l'avait rêvé Murray Bookchin (2003) dans sa vision de l'écologie sociale.

\section{BIBLIOGRAPHIE}

Bardin, L. (1998). L'analyse de contenu. Paris : Presses Universitaires de France. Bookchin, M. (2003). Qu'est-ce que l'écologie sociale ? Lyon : Atelier de création libertaire.

Boulianne, M. (1999). Le potentiel des jardins communautaires pour le développement des collectivités locales : éléments pour l'analyse de l'intervention au Mexique et au Québec, Cahiers du CRISES, numéro ES9910. Consulté le 8 janvier 2010 sur http://www.crises. uqam.ca/cahiers/ ES9910.pdf

Boulianne, M. (2001). L'agriculture urbaine au sein des jardins collectifs québécois : Empowerment des femmes ou "domestication de l'espace public" ? Anthropologie et Sociétés, 25(1), 63-80.

Boutin, G. (1997). L'entretien de recherche qualitatif. Sainte-Foy : Presses de l'Université du Québec. Bouvier-Daclon, N. (2001). La dynamique sociale entourant les jardins communautaires : l'individu, le groupe et le jardin : le cas de Montréal. Thèse de doctorat inédite. Montréal : Institut national de recherche scientifique. 
Bouvier-Daclon, N. et Sénécal, G. (2001). Les jardins communautaires de Montréal : un espace social ambigu, Loisir et société, 24 (2), 507-531.

Breton, R. (1983). La communauté ethnique, communauté politique. Sociologie et sociétés, 15(2), 23-38.

Brunon, H. et Mosser, M. (2007). L'enclos comme parcelle et totalité du monde : pour une approche holistique de l'art des jardins. Ligeia. Dossiers sur l'art, 73-76 (janvier-juin 2007), 59-75.

Chevrier, M. (2001). L'agriculture urbaine ou les paysages nourriciers de la ville. L'Agora, 8(3), 37-39.

Clément, G. (2004). Manifeste du tiers paysage. Paris : Éditions Sujet/Objet.

Courville, A. (2008). L'empowerment dans les jardins collectifs du Québec. Essai de maîtrise inédit. Québec : Université Laval.

Deelstra, T. et Girardet, H. (2000). Urban agriculture and sustainable cities. Dans Bakker, N. et al. Growing Cities, Growing Food (43-65). Feldafing : Deutsche Stiftung für Entwicklung.

Duchemin, É. et al. (2009). Urban agriculture : multi-dimensional tools for social development in poor neibourghoods. Field. Actions Sciences Repertory, 2, 1-8.

Foucault, M. (1967). Des espaces autres (conférence au Cercle d'études architecturales, 14 mars 1967). Architecture, Mouvement, Continuité, 5, 46-49.

Fortin, A. (1987). L'observation participante : au cœur de l'altérité. Dans Deslauriers, J.-P., Les méthodes de la recherche qualitative (p. 23-33). Sillery : Presses de l'Université du Québec.

Freire, P. (1974). Pédagogie des opprimés. Paris : Maspero.

Geoffrion, P. (1997). Le groupe de discussion. In Gauthier, B. (dir.), Recherche sociale : de la problématique à la collecte des données (303-328). Sainte-Foy : Presses de l'Université du Québec.

Goffin, L. (1999). L'environnement comme éco-socio-système. In Loriaux, M. Populations et développement : une approche globale et systémique (p. 199-230). Louvain-la-Neuve/Paris : AcademiaBruylant/L'Harmattan.

Halweil, B. (2002). Home Grown : The Case for Local Food in a Global Market, Worldwatch Paper \# 163. Washington : Worldwatch Institute.

Heller, C. (2002). Désir, nature et société. L'écologie sociale au quotidien. Montréal : Éditions Écosociété.

Henning, J. (1997). Cities Feeding People : an Overview. Communication présentée lors du Forum Cities Feeding People, a Growth Industry, organisé par le Centre de recherche pour le développement international, Ottawa et Montréal, 21-22 mai, 1997.

Hista, J. (2007). Enjeux autour de l'agriculture urbaine contemporaine. Mémoire publié au nom du Regroupement des Jardins Collectifs du Québec, dans le cadre de la Commission sur l'avenir de l'agriculture et de l'agro-alimentaire québécois (CAAAQ), Consulté en ligne le 7 janvier 2010 : http://www.caaaq.gouv.qc.ca/userfiles/File/MEMOIRE/13-12-L-Jardins_collectifs.pdf.

Lavoie, L. (2001). Utopia : De quelques utopies à l'aube du $3^{\mathrm{e}}$ millénaire. Québec : Les Presses de l'Université Laval.

Lebedeva, J. (2008). Climate change adaptation and mitigation through urban agriculture : A Montréal case study. Essai de maîtrise inédit. Montréal : Université McGill.

Lessard-Hébert, M., Goyette, G. et Boutin, G. (1990). Recherche qualitative : fondements et pratiques. Montréal : Agence d'Arc. 
Mahuziès-Sanuy, P. (2007). Jardin et jardinage : un terreau fertile pour la formation continue des enseignants au primaire. Mémoire de maîtrise inédit. Montréal : Université du Québec à Montréal.

Mahuziès-Sanuy, P. (2009), Valeurs jardini'ERE ! Le jardin dans une perspective d'éducation relative à l'environnement. Éducation relative à l'environnement - Regards, Recherches, Réflexions, 8 , 227-233.

McMichael, P. (2001). The Impact of Globalization. Free Trade and Technology on Food and Nutrition in the New Millenium. Proceedings of the Nutrition Society, 60, 215-220.

Mougeot, Luc J. A., (2006). Cultiver de meilleures Villes : agriculture urbaine et développement durable. Ottawa : Centre de recherches pour le développement international.

Negri, T. (discussion avec Petcou, C., Petrescu, D. et Querrien, A.) (2008). Qu'est-ce qu'un événement ou un lieu biopolitique dans la métropole? Multitudes, 31, 17-30.

Nestle, M. (2003), Food Politics, University of California Press, Berkley, LA.

Nourrir Montréal (2009). Réintroduire l'agriculture au cœur des villes : Cultiver des citoyens et des quartiers en santé. Document de référence pour les participants à la Charrette interuniversitaire du Centre canadien d'architecture 2009 - Nourrir la ville. Consulté le 7 janvier 2010 sur http:// www3.cca.qc.ca/charrette/2009/docs/5-NM-document.pdf

Petcou, C. et Petrecu, D. (2008). Agir l'espace : notes transversales observations de terrain et questions concrètes pour chacun de nous. Multitudes, 1(31), 101-114.

Pollan, M. (2006). The Homnivore's Dilemma. New York : The Penguin Press.

Ressources naturelles Canada (2008). Géopanorama de Montréal. Consulté le 15 mai 2010 sur http:// geopanorama.rncan.gc.ca/montreal/soil_f.php

Reyburn, S, (2006). Évaluation de la contribution de l'agriculture urbaine communautaire montréalaise à l'amélioration du cadre de vie. Thèse de doctorat inédite. Montréal : Université du Québec à Montréal.

Rhibes, A. (2006). Toucher la terre. Jardiner avec ceux qui souffrent. Paris : Médicis.

Sauvé, L. (2009). Le rapport entre éthique et politique : un enjeu pour l'éducation relative à l'environnement. Éducation relative à l'environnement - Regards, Recherches, Réflexions, 8, 147-162.

Sauvé, L. (2006). Éducation relative à la santé environnementale : Fondements et pratiques liés à la problématique de l'alimentation en contexte d'éducation populaire et communautaire. Consulté le 7 janvier 2010 sur http://www.eco-alimentation.uqam.ca/

Sauvé, L. (1997). Pour une éducation relative à l'environnement. Montréal : Guérin.

Sauvé, L., et Orellana, I. (2008). Conjuguer rigueur, équité, créativité et amour : l'exigence de la criticité en éducation relative à l'environnement. Éducation relative à l'environnement - Regards, Recherches, Réflexions, 8, 7-20.

Sauvé, L., Panneton, F. et Wojciechowska, M. (2003). L'environnement : de la représentation au concept. Module 2. Programme d'études supérieures - Formation en éducation relative à l'environnement - Francophonie internationale. Montréal : Les Publications ERE-UQAM, Université du Québec à Montréal - Collectif ERE-Francophonie.

Sauvé, L. et Villemagne, C. (2003). L'animation : une dynamique d'éducation relative à l'environnement. Module 17. Programme d'études supérieures - Formation en éducation relative à l'environnement - Francophonie internationale. Montréal : Les Publications EREUQAM, Université du Québec à Montréal - Collectif ERE-Francophonie. 
Sauvé, L., Villemagne, C. et Orellana, I. (2003). Éléments d'une pédagogie de l'éducation relative à l'environnement. Module 4. Programme d'études supérieures - Formation en éducation relative à l'environnement - Francophonie internationale. Montréal : Les Publications ERE-UQAM, Université du Québec à Montréal - Collectif ERE-Francophonie.

Smit, J., Ratta, A. et Nasr, J. (1996). Urban agriculture : food, jobs and sustainable cities. Washington : United Nations Development Programme.

Stiegman, M. (2004). Au cœur de notre quartier; un guide pratique pour le démarrage et l'animation d'un jardin collectif. Montréal : Action Communiterre.

Swartz, M. et Adline, E. (1968). Local-Level Politics : Social and Cultural Perspectives. Chicago : Adline. Thomashow, M. (1995). Ecological Identity : Becoming a Reflective Environmentalist. Cambridge : MIT Press.

Ville de Montréal (2006). La population de Montréal. Montréal en statistiques. Consulté le 15 mai 2010 sur http://ville.montreal.qc.ca/

Villemagne, C. (2005). L'éducation relative à l'environnement en milieu communautaire urbain : un modèle théorique en émergence enrichi de l'exploration collaborative de pratiques éducatives. Thèse de doctorat inédite (cotutelle). Montréal : Université du Québec à Montréal ; Rennes : Université de Rennes 2.

\section{NOTES}

1. L'éducation non formelle concerne l'éducation en milieu non institutionnel (scolaire, gouvernemental) et est souvent portée par les groupes d'éducation populaire et communautaires.

2. Le programme de jardinage communautaire de la Ville de Montréal met à la disposition des citoyens de Montréal 8500 parcelles individuelles réparties dans 95 jardins.

3. Par hétérotopies, concept venant du Grec topos, "lieu », et hétéro, "autre », Foucault entend que le jardin fait partie de ces espaces autres, qu'il appelle aussi les contre-espaces, incarnant physiquement l'utopie et existant en opposition ou en contestation à d'autres sites réels qui se trouvent dans une culture (Lavoie, 2001, p. 61).

4. Gilles Clément est très connu en France en tant que créateur du parc André Citroën et du jardin du musée du quai Branly à Paris.

5. Le jardin ÉCObox est né de l'initiative d'architectes, d'urbanistes et d'étudiants qui décidèrent en 2002 d'investir une ancienne zone industrielle, la Halle Pajol, pour en faire un lieu de rencontres, de création et d'animations autour de l'écologie.

6. La co-responsabilisation signifie la répartition des rôles, des fonctions et des tâches entre les participants afin de favoriser l'engagement des participants dans l'action et l'appropriation du projet par ces derniers (Sauvé et Villemagne, 2003, p. 35).

7. Paulo Freire est un pédagogue brésilien ayant marqué la pédagogie dans la seconde moitié du $\mathrm{XX}^{\mathrm{e}}$ siècle. Il conçoit l'éducation comme un processus de conscientisation et de libération. Son livre phare est sans nul doute Pédagogie des opprimés (1974), qui expose ses idées relatives à l'alphabétisation, à l'éducation des adultes et à l'aspect politique de l'éducation.

8. Duchemin et al. (2009), dans leur article Urban agriculture: multi-dimensional tools for social development in poor neibourghood, présentent une recension d'écrits de ce qu'ils appellent les différentes sphères d'intervention et d'évaluation de l'agriculture urbaine en terme de développement économique, social et environnemental. Les auteurs mettent également en lumière les dimensions éducatives, sociales et productives et ces projets. 
9. De l'anglais « horticultural therapy », le mot « hortithérapie » signifie l'utilisation du jardinage comme support à l'atteinte d'objectifs en santé physique ou mental, ainsi que comme méthode de réinsertion dans la communauté sociale. (Rhibes, 2006, p. 11).

\section{RÉSUMÉS}

Cet article présente une analyse des résultats d'une étude portant sur la caractérisation de l'éducation relative à l'éco-alimentation au sein des jardins collectifs urbains. Cette dernière fut réalisée dans le cadre d'un projet de recherche de la Chaire de recherche du Canada en éducation relative à l'environnement de l'Université du Québec à Montréal. Basée sur deux études de cas menées dans des quartiers aux réalités contrastées de Montréal, cette recherche a permis d'explorer ces initiatives du milieu communautaire en tant que projets d'éducation à l'écoalimentation très ancrés dans le lieu et l'action. La production alimentaire y est centrale, devenant une porte d'entrée privilégiée pour l'apprentissage du vivre-ensemble, l'acquisition de valeurs communes et des principes à la base d'une éco-alimentation. En participant, même à petite échelle, à la reconstruction des relations individus-communauté-environnement au cœur des quartiers où ils sont localisés, les jardins collectifs deviennent des projets éducatifs comportant des dimensions critiques et politiques.

This article discusses the results of a characterization study about Eco-Food Education in urban collective gardens setting. The study was part of a research project undertaken by the chaire de recherche du Canada en éducation relative à l'environnement (Université du Québec à Montréal). Based on two case studies realized in neighbourhoods presenting different realities, the research explored the Eco-Food Education dimension of collective garden initiatives. Food production in collective gardens is a privileged occasion to build shared values and learn about living together and following Eco-Food principles. By participating, even on a small scale, in the reconstruction of the "individual-community-environment" relationships in their respective neighbourhoods, collective gardens become education projects embodying critical and political dimensions.

\section{AUTEUR}

\section{ANNE-MARIE LEGAULT}

Étudiante à la maîtrise en sciences de l'environnement à l'Université du Québec à Montréal, elle s'intéresse aux jardins collectifs comme espace politique et éducatif dans la cité. Passionnée des questions de participation citoyenne, de développement local et de réappropriation des pouvoirs à l'échelle des communautés, elle travaille depuis plusieurs années à la valorisation et à la promotion de l'agriculture urbaine dans la perspective de contribuer à de telles transformations au cœur des villes. 